On June 19th, 2000, the McGill Chapter of Phi Delta Epsilon medical fraternity had the honor of hosting Dr. Miguel N. Burnier Jr., Chairman of the Department of Ophthalmology, McGill University, as the Aaron Brown Lecturer. Dr. Burnier gave a lecture titled "A Story", through which he communicated an inspiring message, and answered this unasked question. "What makes a good physician is not the knowledge one possesses, but three things: ideals, passion, and courage."

Ideals give direction to our lives. It is the ideal of wanting to help the sick that brought most of us to the doors of the medical profession. Through medicine we hoped to cure disease and thus alleviate suffering. However, the suffering of a patient is more than the symptoms of the disease, it is the consequences of the disease - physical, emotional, psychological, and social consequences. Only if we are able to recognize the distinction between disease and illness and address the full impact of both can we alleviate the suffering.

Passion empowers ideals. The practice of medicine in the 21 st century is a foreboding challenge. Physicians are inundated with increased numbers of patients and concomitant decreased availability of support staff and health care funding. These factors not only compromise patient care directly but they also affect the physician's interactions with patients. Physicians, when working in stressful situations, rarely have enough time to spend with their patients. They are unable to provide the holistic care that is needed to alleviate the suffering. Furthermore, these behaviors and stressors are passed down to the residents and the medical students. The passion that we have as young medical students starts to decline as we face the similar challenges of worsening working conditions. The small, yet frequent difficulties we encounter on the way to becoming the "good doctors" we set out to become, make us question the realism of such an entity. Passion empowers us to practice our ideals and the loss of passion allows us to compromise our ideals.

It is courage that will carry us through the difficult times that we may encounter as health care professionals. Courage is the capacity to suffer in the name of our ideals. When in situations that challenge our ideals and dampen our passion, it is courage that sustains us. Courage transforms the challenges we experience into opportunities to grow through suffering. As medical students develop courage, they give depth to their passion and ideals, and thus mature to become the role models they once followed. Thus, young medical professionals need to remember their ideals, sustain their passion, and harness their courage to achieve their goal.

Rina Bansal, B.A., B.Sc., M.D.C.M.

Toni Nimeh

Faculty of Medicine, McGill University
RESEARCH LETTER

\section{TOWARDS AN IMPROVED AIDS VACCINE: ENHANCING THE MHC CLASS I AND II ANTIGEN PRESENTATION OF SIV PROTEINS USING UBIQUITIN AND HLA-DM TARGETING SEQUENCES}

\section{Dear MJM:}

Today, 17 years after the discovery of the human immunodeficiency virus (HIV) and in spite of the considerable effort and funds invested in basic research, acquired immunodeficiency syndrome (AIDS) is still a major epidemic without a cure (1). It is estimated that as many as 40 million people in the world are currently infected. Although numerous advances have been made in understanding HIV biology and pathogenesis and devising treatments, today's clinical therapy is far from solving the underlying pathological problem. The available therapeutic drugs include reverse transcriptase inhibitors and protease inhibitors, which are able to lower viral load to undetectable levels and which seem to improve survival of HIV-infected individuals. This therapy, however, is extremely expensive, has many side effects, and does not prevent a viral rebound after drug withdrawal.

The generation of an effective and affordable vaccine against AIDS is a major goal for developing countries where costly treatment is not an option. Several candidate vaccines have been designed and are currently being tested. One possible approach is the generation of pure DNA vaccines that would import antigenic viral protein into human cells. The proposed mechanism of action is that the foreign protein gets either phagocytosed by, or expressed from within, an antigen-presenting-cell (APC). Antigen presentation may occur by degradation via the ubiquitin-proteosome pathway, association with the major histocompatibility complex class I (MHC I) polypeptide and presentation on the cell surface to $\mathrm{CD} 8^{+}$ T cytotoxic cells; or by degradation and processing in the endosomal/lysosomal compartments, association with MHC II molecules via the endosomal HLA-DM chaperones, and presentation to $\mathrm{CD}^{+} \mathrm{T}$ helper cells, which consequently initiate either a cytotoxic or a humoral response to the antigen.

In a recent collaborative study involving the laboratory of Dr. Franchini at the NCI, NIH, a new highly attenuated NYVAC (New-York-vaccinia virus) vaccine was assessed in Rhesus macaques, which are used as host models for HIV infection. This vaccine contained the gag, pol and env genes (encoding the viral capsid, polymerase, and envelope proteins 
a

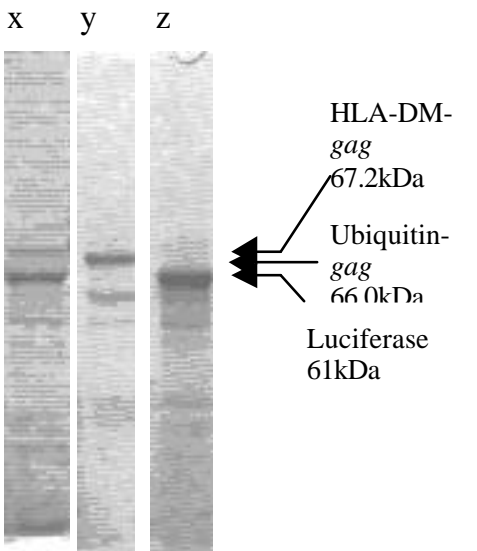

b

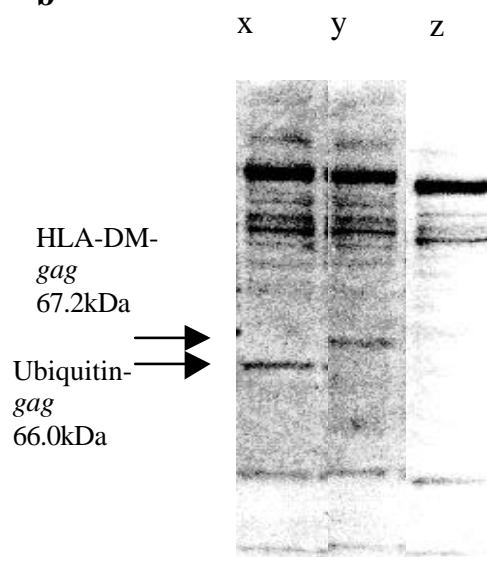

Figure 1. $a$. Detection of radioactive translated proteins ubiquitin-gag (lane x), HLA-DM-gag (lane y) and luciferase, the positive control (lane $\mathrm{z}$ ). $b$. Immunodetection of ubiquitin-gag protein (lane $\mathrm{x}$ ) and HLA-DM-gag protein (lane y). Lane $\mathrm{z}$ contains luciferase, the negative control for immunodetection.

resepectively) of the simian immunodeficiency virus (SIV). Fifty percent of vaccinees were protected against infection with pathogenic SIV virus (2).

Although the partial protection observed in the latter study is encouraging, it remains far from the desired efficiency. Thus, the goal of current research in Dr. Franchini's laboratory is to improve the efficiency of the SIV vaccination protocol, which, if proved successful, may be applied to create an equivalent HIV vaccine. The first component of this goal is priming of the immune system with DNA constructs containing the SIV genes prior to a boost with recombinant NYVAC vaccine. A similar vaccine approach has recently shown promise in a mice model of malaria infection (3). The second component of the goal is improvement of SIV antigen presentation to $\mathrm{T}$ cells via MHC I and II molecules, in view of generating stronger immune responses. This project has adopted two new strategies to enhance the SIV DNA vaccine.

The first strategy is to improve antigen presentation to $\mathrm{CD}^{+}$cytotoxic $\mathrm{T}$ cells via MHC I molecules. The antigenic protein will contain a ubiquitin sequence on the N-terminus causing polyubiquitinylation and targeting to proteosomes for proteolysis and association with the MHC I polypeptide. Ubiquitinylation of protein antigens has been shown to result in decreased stability and induction of cytotoxic lymphocyte responses characterized by higher precursor proliferation and protection against infecting agent $(4,5)$.

The second strategy is to improve antigen presentation to $\mathrm{CD}^{+}{ }^{+} \mathrm{T}$ helper cells via MHC class II molecules. The antigenic protein will contain the signal sequence, transmembrane anchor, and cytoplasmic domain of HLA-DM (6). HLA-DM is a chaperone of the endosomal pathway responsible for antigen-MHC
II molecule association (3). Its localization sequences should target the antigenic SIV protein into the same endosomal compartments $(7,8)$, thus improving the likelihood of its attachment to the MHC II molecules. The efficacy of this approach has been demonstrated in a recent report where the lysosomal targeting sequences of the lysosomalas sociated-membrane-protein-1 (LAMP-1) were used in conjunction with a human papillomavirus antigen. The fusion protein was successfully targeted to endosomal/lysosomal compartments and was able to elicit a much higher cell-mediated immune memory response in mice than antigen alone (9).

For both of the above strategies, an expression cassette was created using the given targeting sequences and containing specific restriction sites, which would allow the insertion of any gene, such as gag (used in this project).

The ubiquitin sequence (259bp), the HLA-DM signal, connecting, transmembrane and cytoplasmic domains (293bp), and the gag sequence (1573bp) were successfully amplified by PCR from the macaque genomic DNA and a CMVkan/gag plasmid. The primers used to amplify ubiquitin (10) and HLA-DM (6) sequences were designed according to the human gene, since these sequences appear to be highly conserved among species. The primers contained specific restriction sites that served to ligate targeting sequences into a pGEM $3 \mathrm{zf}^{+}$vector, optimized for in vitro translation. The resulting plasmids were amplified via transformation of bacterial $E$. coli cells.

To verify the proper sequence of the genetic constructs, the plasmids pGEM $3 \mathrm{zf}^{+}$(negative control),

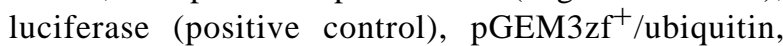
pGEM3zf ${ }^{+} /$HLA-DM, pGEM $3 \mathrm{zf}^{+} /$ubiquitin-gag and pGEM5zf/HLA-DM-gag were translated in vitro using the TNT Coupled Reticulocyte Lysate System kit (Promega), in presence of radioactive ${ }^{35} \mathrm{~S}$-methionine. The fusion proteins ubiquitin-gag (predicted size 66.0 $\mathrm{kDa})$ and HLA-DM-gag (67.2 kDa) were analyzed by SDS-polyacrylamide gel electrophoresis, and the expected bands were seen after detection on X-ray film (Figure 1A). The same proteins were separated on another gel and analyzed by Western blot with anti-SIV-gag antibody. This specific immunodetection showed the ubiquitin-gag protein at 66.0 $\mathrm{kDa}$ and the HLA-DM-gag protein at $67.2 \mathrm{kDa}$ (Figure 1B). 

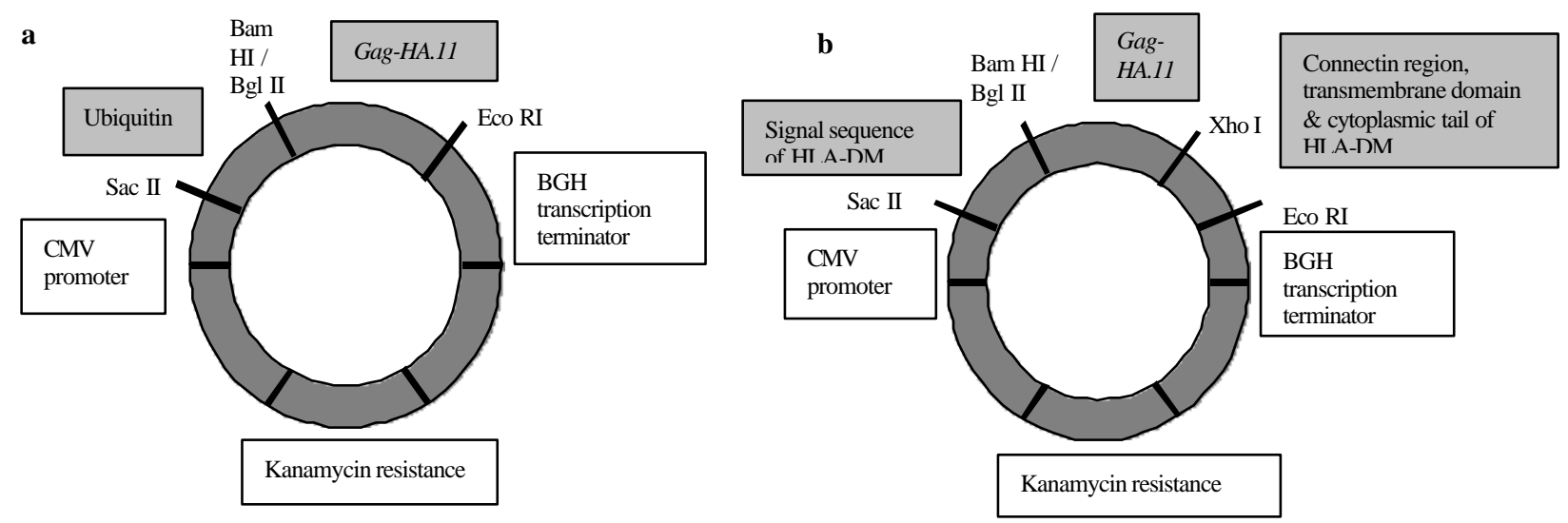

Figure 2. Schematic diagrams for the $a$. CMVkan/ubiquitin-gag plasmid and $b$. CMVkan/HLA-DM-gag plasmid.

The final constructs, ubiquitin-gag and HLA-DMgag, were excised from the pGEM3zf+ vectors and cloned into CMVkan vector within the unique SacII and EcoRI restriction sites, downstream of the CMV promoter (optimizing expression in eukaryotic cells) and upstream of the $\mathrm{BGH}$ transcription terminator sequence (Figure 2).

The project described herein succeeded in creating two chimeric constructs, both containing the SIV gag gene as a prototype of an SIV viral antigen. The first construct contains the ubiquitin sequence and the second construct contains the $\mathrm{N}$-terminal signal sequence, as well as the $\mathrm{C}$-terminal targeting regions of HLA-DM. These constructs were first inserted into a pGEM vector, in order to perform an in vitro translation. This translation was crucial in the development of the project, because it confirmed the sequences amplified by PCR, and the in-frame insertion of all the genes. It ruled out possible out-offrame deletions or insertions, which would change the reading frame of the genes or the target sequence detected by the anti-SIV-gag antibody. Nevertheless, point mutations may still be left undetected within the constructs. Therefore, sequencing of the whole constructs is to be performed.

The genetic constructs ubiquitin-gag and HLA-DMgag were cloned into a CMVkan vector, which is optimized for expression in eukaryotic cells. This vector would ultimately be used in vivo as the DNA vaccine. However, the efficacy of the engineered CMV vectors first has to be determined in vitro. Several experiments are presently planned to analyze the in vitro functionality of the CMV/ubiquitin-gag and CMV/HLA-DM-gag plasmids, using the CMV/gag plasmid and the CMV vector alone as negative controls.
The first step will be transfection of the plasmids into human cell line (Hela). The gag protein has been generated in such a way that the C-terminus contains the 9 amino-acid recognition sequence tag HA.11 (11). Expression of the recombinant protein will be monitored using antibodies specific to the HA.11 by Western blotting and immunodetection. The expression of CMV/ubiquitin-gag vector will be analyzed in presence or absence of ALLN (a calpain protease inhibitor), which should block its degradation by the proteosome pathway. The intracellular vesicular localization of CMV/HLA-DM-gag vector will be detected in presence and absence of leupeptin (an endosomal protease inhibitor) using anti-HA.11 fluorescent antibody or by a colocalization study using antibody to endogenous LAMP-1 (marker for endosomal/lysosomal compartments) (9).

The final in vitro functional test will be an immunological assay, where peripheral blood mononuclear cells (PBMC) from an infected rhesus monkey will be transfected with increasing concentration of CMV/ubiquitin-gag or CMV/HLA-DM-gag constructs. Specific proliferation of the immune cells in response to the gag antigen will be detected by lymphoproliferative assay. The transfected cells would represent a model of the host immune system primed with a DNA vaccine, and would be expected to proliferate more than the negative controls.

If the functional assays performed with the CMV/ubiquitin-gag and CMV/HLA-DM-gag plasmids are encouraging, an in vivo experiment will be set-up using Rhesus macaques. The primary advantage of these DNA expression cassettes is that the gag antigen used in this study can be easily replaced by other viral antigenic proteins in the course of the study, due to the unique restriction sites. If further positive results are 
obtained, the strategy presented in this report would be applied to HIV vaccines and considered for clinical trials, in hope of providing immunological defense to victims of HIV, and limit further spread of the virus to uninfected individuals.

Tereza Martinu

Faculty of Medicine, McGill University

Zdenek Hel, Ph.D.

Genoveffa Franchini, M.D.

National Cancer Institute, NIH, Bethesda

\section{REFERENCES}

1. Luciw PA. Human immunodeficiency viruses and their replication. In: Fields BN, Knipe DM, Howley PM, eds. Fields Virology. Philadelphia: Lippincott-Raven Publishers; 1996.

2. Benson J, Chougnet C, Robert-Guroff M, et al. Recombinant vaccine-induced protection against the highly pathogenic simian immunodeficiency virus SIV $_{\text {mac251: dependence on }}$ route of challenge exposure. Journal of Virology 1998; 72: 4170-4182.

3. Schneider J, Gilbert SC, Blanchard TJ, et al. Enhanced immunogenicity for $\mathrm{CD} 8+\mathrm{T}$ cell induction and complete protective efficacy of malaria DNA vaccination by boosting with modified vaccinia virus Ankara. Nature Medicine 1998; 4: 387-402.

4. Michalek MT, Grant EP, Gramm C, et al. A role for the ubiquitin-dependent proteolytic pathway in MHC class Irestricted antigen presentation. Nature 1993; 363: 552-554.

5. Rodriguez F, Zhang J, Whitton JL. DNA immunization: ubiquitination of a viral protein enhances cytotoxic $\mathrm{T}$ lymphocyte induction and antiviral protection but abrogates antibody induction. Journal of Virology 1997; 71: 8497-8503.

6. Kelly AP, Monaco JJ, Cho S, Trowsdale J. A new human HLA class II-related locus, DM. Nature 1991; 353: 571-573.

7. Lindsted R, Liljedahl M, Peleraux A, et al. The MHC class II molecule H2-M is targeted to an endosomal compartment by a tyrosine-based targeting motif. Immunity 1995; 3: 561-572.

8. Marks MS, Roche PA, van Donselaar E, et al. (1995) A lysosomal targeting signal in the cytoplasmic tail of the $b$ chain directs HLA-DM to MHC class II compartments. Journal of Cell Biology 1995 131: 351-369.

9. Wu T-C, Guarnieri FG, Staveley-O'Carroll KF, et al. Engineering an intracellular pathway for major histocompatibility complex II presentation of antigens. Immunology 1995; 92: 11671-11675.

10. Baker RT, Board PG. The human ubiquitin gene family: structure of a gene and pseudogenes from the Ub B subfamily. Nucleic Acids Research, 1987; 15: 443-463.

11. Ausbel FM, et al., eds. Current Protocols in Molecular Biology. New York: Greene Publishing Associates and WileyInterscience; 1987 (updated bimonthly). 